\title{
Research on evaluating the performance of protective coating based on Electrochemical Impedance Spectroscopy
}

\author{
Liwei PENG ${ }^{1, \text { a }}$,Zhongju ZHANG ${ }^{2, \text { b }}$ \\ ${ }^{1}$ Department of Driver Training Service, Bengbu Automobile NCO \\ Academy, Bengbu, 233011, China \\ ${ }^{2}$ Department of Driver Training Service, Bengbu Automobile NCO \\ Academy, Bengbu, 233011, China \\ axiulianshi@163.com, ${ }^{b} 774851579 @ q q . c o m$
}

\begin{abstract}
Researching on the performance of protective vehicle's coating. The results manifest that the coating's performance is different from each other, even surface is perfect. Carbon steel sheets coated with organic coating with various degrees of simulated damages are electrochemically researched in $\mathrm{NaCl}$ solution. The parameters of corresponding coating system are presented. These parameters provide a clue on theory for developing field testing. The EIS of organic coatings of various damages were acquired and analyzed.
\end{abstract}

Keywords: Coating; Evaluating; EIS (Electrochemical Impedance Spectroscopy)

\section{Introduction}

One of the main failure forms of vehicle's organic coating is broken in actual service. Because of scratch and mechanical damage in service, the corrosive medium easily invade from broken coating, then corrosion resistance of coating will be worse. Researchers had explored from various aspects, and had made many satisfactory results, but which almost had taken place in ideal states of coating. And the research focus on evaluating corrosion resistance of organic coatings, very few researches taking place in actual working condition had been reported. This paper simulated different damage degree of coatings, which will be broken in service, then made electrochemical test in $3.5 \% \mathrm{NaCl}$ solution, revealed the characteristics of damage process, explored the influence factors, provided a theoretical basis for on-the-spot detection of damage of organic coating[1,2,3,4,5] 


\section{EIS of the performance of protective coating}

Testing coating is alkyd paint, finishing coat is acrylic amino alkyd enamel. Fig. 1 are probes which are designed to test on-the-spot. The test area is $13 \mathrm{~cm} 2$. $3.5 \% \mathrm{NaCl}$ solution. PRINCETON PARSTAT 2263. String perturbation amplitude of EIS is $10 \mathrm{mV}$. Frequency is $10-2 \mathrm{~Hz} \sim 10 \mathrm{kHz}$, logarithmic scan, 8 steps. Using three electrode system. The auxiliary electrode is titanium. The reference electrode is SCE. The working electrode is body of vehicle. 22 . $\mathrm{C}$ Mapping by Origin7.0 software, Data analysis by Zsimpwin software.

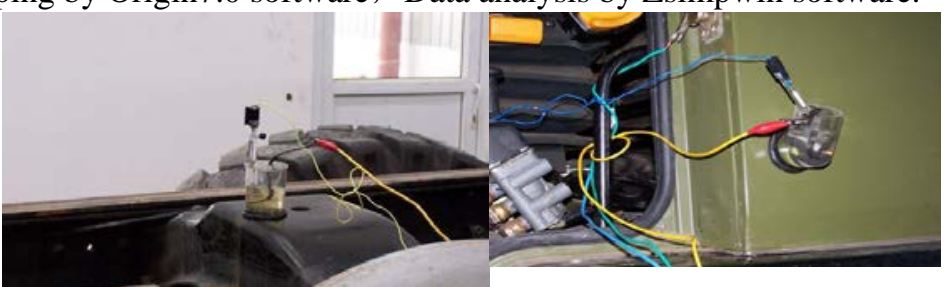

Fig.1 Probe adapting to on-the-spot test

We can make a conclusion that the coating has apparent defect by EIS test, which appears perfect from the it's surface in Fig.2. Electrolyte solution had reached coating and formed the corrosion reaction micro battery. There are two time constants and Warburg impedance in Nyquist, which may be blocked by additives in coating. Electrolyte solution immersed particles and permeate deeply, caused the Tangential diffusion phenomenon.
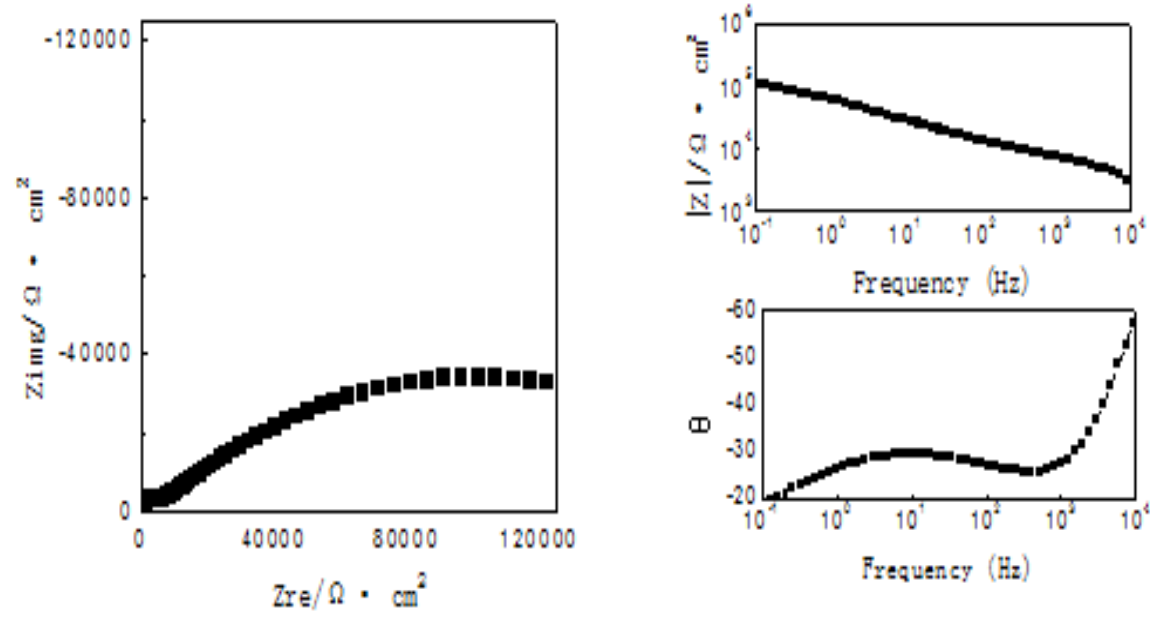

Fig.2 EIS of slight defects coating

Fig. 3 is EIS test of serious defects coating. Warburg impedance is obviously, which means electrolyte solution can easily immersed the base metal from the broken area. Coating has gradually lost protective effects. 

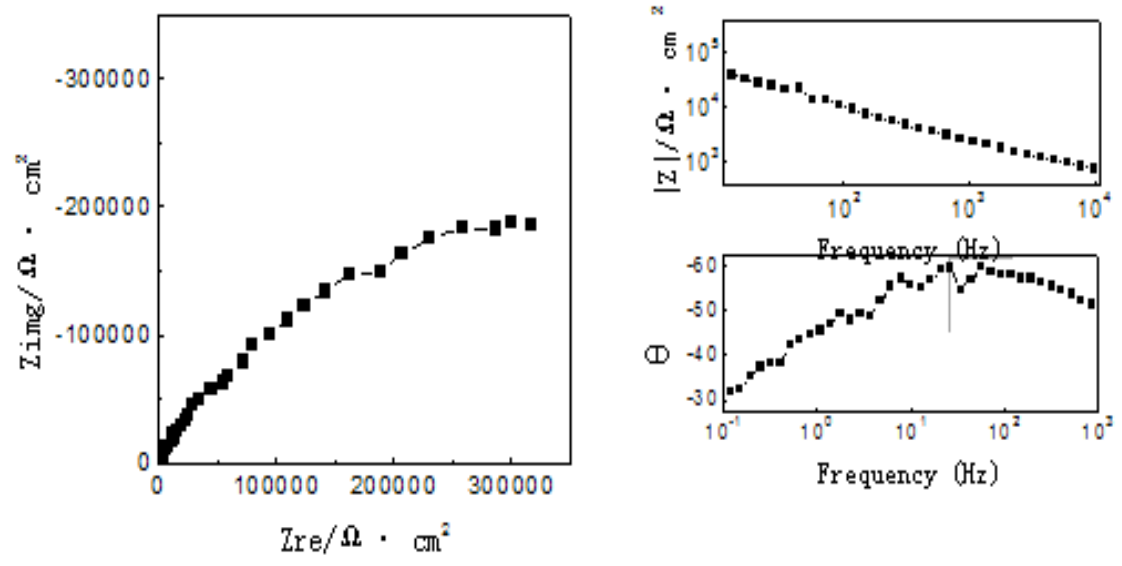

Fig.3 EIS test of seriously defects coating

\section{Simulation study on coatings with different degree of damage}

According to the characteristics of coating damage, design sample 1\#、2\#, thickness is $90 \mu \mathrm{m}$, broken diameter with $\Phi 1=0.90 \mathrm{~mm}, \Phi 2=1.28 \mathrm{~mm}$, $\Phi 3=1.82 \mathrm{~mm}, \quad \Phi 4=2.58 \mathrm{~mm}, \Phi 5=2.88 \mathrm{~mm} .3 .5 \% \mathrm{NaCl}$ solution.
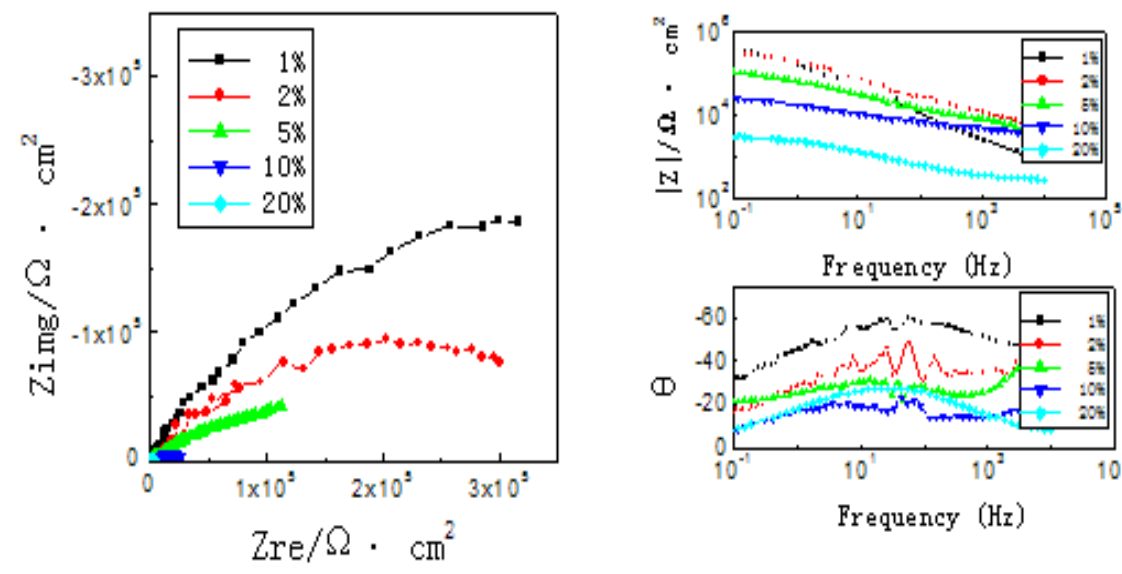

Frequency $\left(\mathrm{H}_{z}\right)$

$(1$

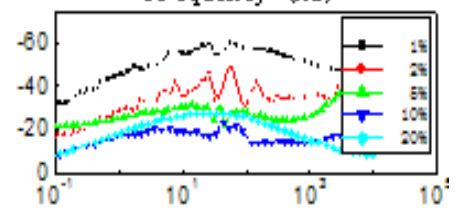

Frequency $(\mathrm{Hz})$

Fig.4 EIS test of different broken coatings

Fig.4 is EIS test of different broken coatings. Single capacitive arc appears in EIS, which coating is perfect. With the broken seriously, double capacitive arcs appear in EIS, which means two time constants. Characteristics of coating was presented in low frequency region. Characteristics of metal covered by coating was presented in high frequency region. Impedance decreases with broken diameter of coating increasing. Inductive reactance appears in EIS with $10 \%$ 
broken rate of coating, which means corrosion accelerating with metal covered by coating. Coating has completely lost the protective effect with $20 \%$ broken rate of coating, which appears contraction in real part.

Capacitive reactance of coating is high when signal frequency is low. Rs can be ignored. Fig. 5 is equivalent circuit.

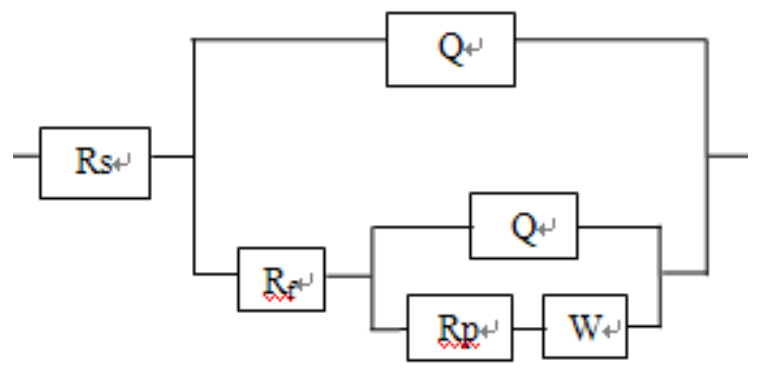

(a)

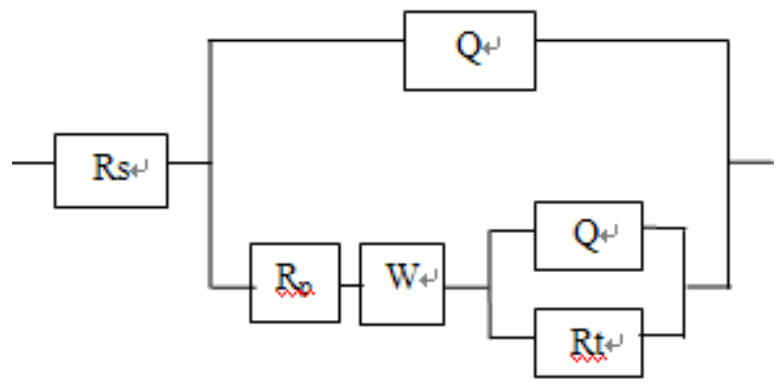

(b)

Fig.5 Equivalent circuit of broken coating

EIS was fitted by ZSimpWin software. Coating of $\Phi 1 \sim \Phi 4$ are fitted with equivalent circuit of Fig.5 (a) .Coating of $\Phi 5$ is fitted with equivalent circuit of Fig.5 (b) . Fig.6 is the fitting results of $5 \%$ broken rate of coating. Table1 is the fitting results.

Definition of coating broken coefficient K:

$\mathrm{K}=\mathrm{S}_{\mathrm{p}} / \mathrm{S}$.

(1)

Sp: the acreage of broken area of coating. S: the acreage of test piece.

Rf: coating resistance. Rp: polarization resistance

Table 1 fitting results

Num. broken diameter, cm $\mathrm{K} \quad R_{p}, k \Omega c m^{2}$




\begin{tabular}{cccc}
\hline 1 & 0.41 & $1 \%$ & $5.49 \mathrm{E} 3$ \\
534.6 & & & \\
2 & 0.57 & $2 \%$ & $4.01 \mathrm{E} 3$ \\
8.90 & & $5 \%$ & $1.09 \mathrm{E} 3$ \\
3 & 0.91 & $10 \%$ & 98.38 \\
5.32 & & & \\
4 & 1.29 & $20 \%$ & 55.72 \\
4.29 & & & \\
5 & 3.63 & & \\
3.25 & & & \\
\hline
\end{tabular}
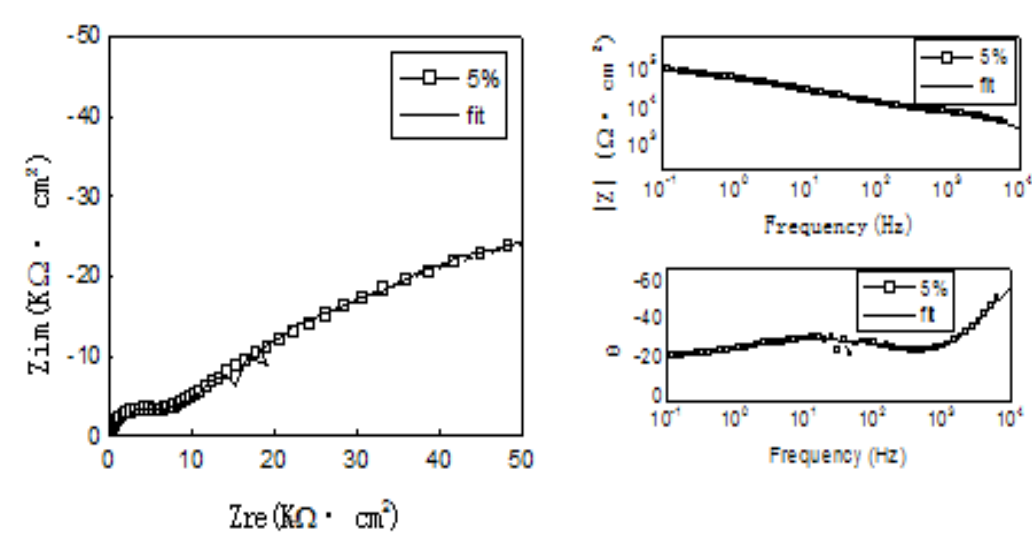

Fig.6 Fitting results of 5\% broken rate of coating

Using the method of regression analysis to fit the change curve of $\mathrm{Rp}, \mathrm{Rf}$ relative to $\mathrm{K}$ in table1, we can get the following function relation:

$\log R_{\mathrm{p}}=0.556-1.639 \log \mathrm{K}, \mathrm{r}^{2}=0.984$.

(2)

$\operatorname{logRf}=0.817-1.438 \log \mathrm{K}, \mathrm{r} 2=0.951$

The correlation coefficient is larger than 0.95, which means results fitting the actual test.

\section{Conclusion}

Designing two kinds of probe adapting to on-the-spot test. With test the EIS of coating and fitting the results of equivalent circuit, we can evaluate the performance of coating on-the-spot. Attempting to put forward a new method, which can be used in evaluating the performance protective coating of vehicles on the field. Bode diagram of EIS on the field measured is disorder, which can't be used to analysis. So we try to use the low steady frequency of the Bode 
diagram, and establish the relationship curve of $|\mathrm{Z}|$ for field and lab measured to evaluate the performance of protective coating of military vehicle.

\section{References}

[1] S. Tung, T. M. Devin:The 202nd Electrochemical Society Meeting, Meeting Abstracts, Sat Lake City, Utah, 2002,G1-G25.

[2] T. L. Han, J. Q. Liu, W. Y. Luo:Mater. Prot. (2005),p. 38, 31.

[3] C. N. Cao: Principle of Corrosion Electrochemistry(Chemistry Industry Press, China, 2004), p. 2-10.

[4] H. Yan:New Techniques in Electroless Nickel and Composite Plating(Industry of National Defense Press, China, 2001),p. 1.

[5] X. X. Jiang, W. Shen, The Fundamental and Practice of Electroless Plating, Defence Industry Press, China,2000),p. 1-10. 